\title{
Uterine Myoma: A Profile of Nepalese women
}

\author{
Pramila Pradhan, Nitish Acharya, Binit Kharel, Manoj Manjin
}

\begin{abstract}
:
Objectives:

1. To determine the most common age and parity for the development of myoma uteri.

2. To find out any relation between age of the last child and the development of myoma uteri.

3. To determine the treatment protocal and outcome of treatment.
\end{abstract}

Materials/methods: This study was carried out at Nepal Medical College Teaching Hospital from Jan 2001 to $31^{\text {st }}$ June 2006. All cases of myoma uteri admitted in Gynaecological ward was included.. Histological evidence of myoma confirmed the diagnosis. Age, parity and age of the last child, clinical features, surgical procedures and outcome of surgery were considered.

Results: A total of 137 cases of myoma uteri was operated during five and half years period. This represented $38.5 \%$ of all abdominal hysterectomy performed during the same period. The mean age was 43.3 years with median parity at 1-3 and the mean age of last child at 12 years. Excessive menstrual bleeding $73.0 \%$, abdominal mass and pain 58.4\%, dysmenorrhea $18.2 \%$ and infertility $7.3 \%$ were the common symptoms. Total abdominal hysterectomy was the mainstay of treatment. Patients were happy with the treatment specially those with severe and multiple symptoms Postoperative complications were not significant. There was one death due to pulmonary embolism who had mesothelioma of pleura diagnosed by pleural biopsy before operation.

Conclusion: Early marriage and early completion of the family is the social trend in Nepalese society. Myoma is common in $4^{\text {th }}-5^{\text {th }}$ decade of life in multi parity with the age of last child around 12 years. This long standing secondary infertility may be an underlying risk factor in the development of myoma uteri.

\section{Introduction}

Uterine myomas are common benign clonal tumours that arise from the smooth muscle cells of uterus. They are clinically apparent in about $25 \%$ of women and with newer imaging techniques and careful examination of surgical specimen suggests that the prevalence is as high as $77 \% .{ }^{1}$ Approximately $60 \%$ of all laparotomies performed on women for pelvic disease are due to myomas and less than $1 \%$ are malignant ${ }^{2}$ Myomas are more common in nulliparous or relatively infertile women. ${ }^{3}$ Race is important epidemiological risk factor for myomas. Black negroid women have over 3 fold greater frequency and greater morbidity. ${ }^{4,5}$ Presently there is increased evidence that myomas have a genetic basis and that their growth is related to genetic predisposition, hormonal influence and various growth factors. ${ }^{6}$ Depending on their size and location, myoma can be completely asymptomatic or can cause excessive menstrual bleeding, pelvic pressure, mass, pain and / or reproductive dysfunction. Uterine myomas are observed in pregnancy more frequently now than in past because many women are delaying child bearing to their late thirties, the time of greatest risk for myoma growth. ${ }^{7}$ Hysterectomy has been the mainstay of treatment in women who completed their family. Several recent publications reported improvement in quality of life for most women who have had hysterectomy and concluded that hysterectomy does not adversely influence sexuality. ${ }^{8,9}$ Myomectomy is frequently done in women who desire to preserve the child bearing function. ${ }^{10-12}$ There are now several operative techniques (endoscopic surgery, uterine artery embolization, high frequency ultrasonography, laser treatment, myolysis, thermoablation) that do not require major surgery and fertility is maintained. ${ }^{6}$ Medical management with hormone like progestational agents, gonadotrophin releasing hormone $(\mathrm{GnRH})$ agonists are described but there is no consensus regarding routine use of these drugs. ${ }^{6}$

This study is carried out to find out the most common age for the development of myoma of uterus, the common parity of women who are suffering from myoma of the uterus, to find out any relation between the age of the last child and the development of myoma of uterus, management protocol and outcome of surgery in Nepalese women. 


\section{Materials and method}

This study was carried out in Gynaecology department in Nepal Medical College Teaching Hospital, Kathmandu, Nepal over a period of five and half year period from Jan 2001 to $30^{\text {th }}$ June 2006. All the patients admitted in gynaecological ward, as a case of myoma of uterus was included in the study. Histopathological evidence of myoma confirmed the diagnosis. Information on the total number of gynaecological cases admitted were obtained from gynaecological ward register and total number of abdominal hysterectomy cases were obtained from operation theater register book. A total of one hundred and forty cases diagnosed clinically as myoma uteri were admitted for surgery. Ultra sonogram was additional tool for diagnosis. CT scan was done in limited cases only. Three cases turned out to be retroperitoneal mass and handed over to surgeons for further management. A total of one hundred and thirty seven cases of myoma uteri were studied to find out, parity, age of last child and development of myoma, clinical presentation, management protocol and outcome of surgery.

\section{Results}

A total of one hundred and thirty-seven cases of myoma uteri were operated in five and half year period. The incidence of myoma was $8 \%$ of all Gynaecological admission and $38.5 \%$ of all abdominal hysterectomy performed in this institution. The age of the patients ranged from 23 to 60 years with mean age of 43.3 years. The commonest age group for the occurrence of myoma is 41 to 50 years $(56.2 \%)$ table I. The parity of the patients ranged from $0-11$, majority were multiparity having children 1-3 (46.7\%).(table I) The age of last child range from 5-25 years with mean of 12 years. Maximum patients having the last child of about 11-20 years age was $60 \%$.(table II) The common symptoms were excessive menstrual bleeding in 100 cases(73.0\%),

Table I: Age and Parity Distribution

\begin{tabular}{lcc}
\hline Age (in yrs) & No & \% ge \\
\hline $20-30$ & 4 & 2.9 \\
$31-40$ & 39 & 28.4 \\
$41-50$ & 77 & 56.3 \\
$51-60$ & 15 & 10.9 \\
$61+$ & 2 & 1.5 \\
\hline Mean age = 43.3 yrs & & \\
\hline Parity & No & \% ge \\
\hline Nulliparity & 14 & 10.3 \\
$1-3$ & 64 & 46.7 \\
$4-6$ & 48 & 35.0 \\
$7-10$ & 10 & 7.3 \\
$11+$ & 1 & 0.7 \\
\hline
\end{tabular}

Median Parity $=1-3$
Table II : Distribution of Age of Last Child

\begin{tabular}{lcc}
\hline Years & No & \% g e \\
\hline $1-5$ & 4 & 3.2 \\
$6-10$ & 23 & 18.9 \\
$11-20$ & 74 & 60.0 \\
$21-25$ & 22 & 17.8 \\
\hline Total & $\mathbf{1 2 3}$ & $\mathbf{1 0 0}$ \\
\hline
\end{tabular}

Table III: Clinical Features

\begin{tabular}{lcc}
\hline Symptons & No & \% g e \\
\hline Menorrhagia & 100 & 73.0 \\
Abdominal Pain & 80 & 58.4 \\
Abdominal Mass & 18 & 13.0 \\
Dysmenorrhea & 25 & 18.2 \\
Infetility & 10 & 7.3 \\
Retension of Urine & 3 & 2.2 \\
\hline Physical Signs & & \\
Abdominal Mass & 117 & 85.4 \\
Anaemia Mass & 25 & 18.2 \\
\hline
\end{tabular}

Severe anaemia(Hb5-7)in 25cases(18.1\%) needing preoperative blood transfusion, abdominal pain in 80 cases $(58.4 \%)$, abdominal mass in 18 cases $(13.0 \%)$, dysmenorrhea in 25 cases $\mathrm{s}(10.9 \%)$, infertility in 10 cases $(7.3 \%)$, urinary retention and asymptomatic at $2.2 \%$ each. Among the physical signs abdominal mass in 117 cases $(85.5 \%)$ and anaemia in 25 cases (18.8\%) were significant (table III Clinical diagnosis was correct in 135 cases $(98.5 \%)$ and ultrasound was the additional tool for diagnosis. Total abdominal hysterectomy with or without salpingo-ophorectomy was the mainstay of surgery. Abdominal myomectomy were done in two cases of infertility (1.4\%). In post operative period oneweek course of antibiotic coverage was the rule in this institution. Histological diagnosis of myoma was confirmed in all cases. Postoperative complications were, minor wound infection in 16 cases $(11.7 \%)$ and 10 cases $(7.3 \%)$ needed resuturing of abdominal wound, urinary tract infection occurred in 10 cases $(7.3 \%)$, minor secondary haemorrhage per vaginum occurred in three cases managed conservatively (Fig 1). Blood transfusion of 1-8 units needed in 52 cases (38\%) Follow up were possible in 100 cases $(73.0 \%)$ for over 1-3 years and 16 cases $(11.7 \%)$ were given hormone replacement therapy with estrogen (conjugase) $0.625 \mathrm{mg}$ orally daily for postmenopausal symptoms (Table 4). All follow up cases were happy with the treatment and felt improved quality of life in day-to-day life and in sexuality.

\section{Discussion}

Uterine myoma is the most common solid pelvic tumour in women occurring in 20-40\% during their reproductive life. Infertile and sub fertile women are affected most commonly ${ }^{3}$. In the present study, it was found that 
Table IV: Post Operative Morbidity

\begin{tabular}{lcc}
\hline Variables & No & \%ge \\
Blood Transfusion $(\mathbf{n = 5 2})$ & & \\
Preoperative & 32 & \\
Postoperative & 20 & \\
1-4 Units & 44 & \\
5-8 Units & 8 & \\
Wound Infection $(\mathbf{n = 2 6})$ & & \\
Minor Wound Infection & 16 & 11.7 \\
Secondary Suturing & 10 & 7.3 \\
Uniraty Tract Infection & 12 & 8.8 \\
Secondary Vaginal & 3 & 2.2 \\
Haemorrhage & & \\
\hline
\end{tabular}

uterine myoma usually occurred in multiparous women, median parity $1-3(46.7 \%)$ most often in the age group of 40-50 years $(56.2 \%)$ and the age of the last child is usually between 10-20 years with average of twelve years. Studies done in different teaching institutes of Bangladesh were similar to the present study except they have significantly high parity of six to seven children (52\%). ${ }^{12-14}$ A study done is Nigeria found that the most common age was 30-39 years with mean of 44.9 years with nullipara at $40.8 \%$ and $35 \%$ women were para 1-2 which were different from this study. ${ }^{15}$ Buttrum and Reiters ${ }^{16}$ reviewed 1698 cases of myoma uteri of which $27 \%$ had history of infertility and $31 \%$ had 1-2 children. Another study done in Catholic University Rome ${ }^{17}$ on pregnant patients and found that the mean maternal age was 31.6 years, $42 \%$ having myoma in their $1^{\text {st }}$ pregnancy, $58 \%$ were in their $2^{\text {nd }}$ pregnancy. From these studies it is clear that myoma uteri is more common in nulliparous or subfertile women in those countries. The age of the last child was found to be in the range of 6-20 years in different studies ${ }^{12-14}$ which is similar to this study at $11-20$ years $(60 \%)$. The long standing secondary infertility both voluntary or involuntary cause continuous oestrogen secretion uninterrupted by pregnancy and lactation which may be an underlying risk factor in the development of myoma uteri. ${ }^{6}{ }^{12}$ Excessive menstrual bleeding was the main symptom at $73.0 \%$ and 25 patients $(18.5 \%)$ of them needed blood transfusion before operation. These findings were similar to other studies. ${ }^{6,12-15}$ Excessive menstrual bleeding an often the only symptom in myoma, is attributed to vascular alterations, increased surface area of the endometrium and increasing size of the uterine cavity and endometritis which is frequently observed histologically in the endometrial tissue overlying the submucous tumours. ${ }^{3,6,7}$ A dysregulation of local growth factors and aberrant angiogenesis has also been implicated in the abnormal bleeding pattern. ${ }^{18}$ Pain in myoma is usually associated with torsion of a pedunculated myoma, by a submucous myoma protruding through the cervical canal or red degeneration in pregnancy. Pain is acute in these conditions and require immediate attention. Pain abdomen was a significant symptom at $58.4 \%$ in this study and is similar to other studies. ${ }^{1,3,6,7}$ six cases $(4.3 \%)$ were presented with submucous myoma protruding through cervix on long pedicle but were not in acute stage needing immediate attention. This may be due to long standing history in these cases. Adenomyosis can cause pain. Magnetic resonance imaging may prove helpful differentiating from myoma. ${ }^{6}$ Pressure and increased abdominal girth are more commonly encountered than pain. As myoma grows pressure is exerted on adjacent viscera with manifestation from the urinary or bowel problems. ${ }^{6,7}$ An increased abdominal mass was seen in $85.5 \%$ in this study and the size varied from 8 weeks to36weeks gestational weeks and the biggest mass removed weighed $10 \mathrm{kgs}$ from an unmarried 36years old lady.

Infertility may be associated with a submucous myoma or a markedly distorted, enlarged endometrial cavity that interfere with normal implantation or with sperm transportation. Severe displacement of the cervix is also capable of adversely affecting sperm deposition at the cervical os or obstruction of tubal ostia or intramural portion of the tube by a large intramural myoma may affect infertility. ${ }^{6}$ Uterine myomas have also been implicated in recurrent pregnancy loss. ${ }^{19}$

Malignant transformation of myomas is extremely rare and felt that leiomyosarcoma arise de novo and may be unrelated to benign myoma. ${ }^{6}$ Different studies ${ }^{6,7,20}$ reported Leiomyosarcoma in between $0.13-0.29 \%$. In postmenopausal women rapid growth must raise the suspicion of a malignancy.

Hysterectomy has been the usual procedure of choice whenever surgery is indicated for uterine myomas who have completed the family. Studies have demonstrated that for most women, hysterectomy is very effective in allevating symptoms and improving the quality of life in the years following surgery. ${ }^{8,9}$ Total abdominal hysterectomy with or without salpingoophorectomy was the mainstay of treatment $(98.5 \%)$ in this study (Fig1) with strong evidence of substantial improvement of symptoms for women with multiple and severe symptoms associated with myoma. We do not

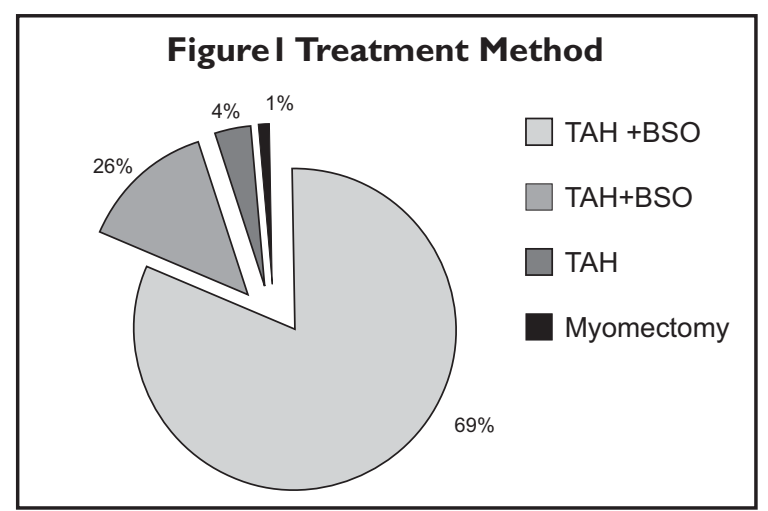


remove the ovaries at the time of surgery for women less than 45 years of age unless there is underlying pathology. Two cases $(4.3 \%)$ of sub fertility had abdominal outcome is awaited. Myomectomy may improve reproductive outcome although there are no prospective studies to prove it. ${ }^{10,11}$ Myomectomy on the other hand is not without a risky procedure like re growth of myoma or safety in subsequent pregnancy. ${ }^{21}$ No other methods of treatment were used in this study.

Hysterectomy for uterine myomas is not free of complications. Anatomic distortion of the uterus increase the risk of damage to adjacent structures. The incidence of ureteral injury in one extensive report was 0.4 per 1000 with total abdominal hysterectomy and that of vesico vaginal fistula was 1.0 per $1000 .^{6}$ The reported overall mortality rate with this surgery is 12 per $100,000 .^{6,15}$ Longterm problems after hysterectomy for myomas include development of vaginal vault prolapse. Supracervical hysterectomy has been proposed to lessen the risk of vault prolapse, for better sexual function and to reduce the risk of urinary tract injury. But a recent prospective randomized study revealed no difference in sexual activity and orgasm or pelvic relaxation symptom after 2 years of follow-up. ${ }^{22}$ No significant post operative complications occurred in this study. Blood transfusion was needed in $38.5 \%$, wound infection in $19.0 \%$, urinary tract infection in 12 cases $(8.8 \%)$. There was one death in this study who had suffered from pleural effusion with pleural mesothelioma diagnosed by pleural biopsy before operation.

\section{Conclusion}

Early marriage and early child bearing is common in our society. The incidence of multiparity is also high. The women usually complete their family by 35 years and enjoy a long pregnancy free time, which is equivalent to secondary infertility, a risk factor for the development of myoma uteri. Removal of uterus is a very satisfactory method of treatment without much morbidity with a substantial improvement of symptoms and improved quality of life.

\section{References}

1. EA Stewart uterine fibroid. Lancet 2001; 357: 29398.

2. Smith charles J. Fibromyoma of uterus; AmJ obstet gynaecol 1952; $64: 1211$.

3. Tendal VR . Jeffcoates's Principle of Gynaecology; fifth edition; Butterworth London ; 1993 ; 419-32.

4. Marshal $1 \mathrm{M}$, Spiegelman D, Barbieri R et al. Variation incidence of uterine leiomyoma among premenopausal by age and race. Obstet Gynaecol $1997 ; 90: 967-73$.

5. Kjeruff KH, Langberg P, Seidman JD, Stolley PD, Guzinski GM. Uterine leiomyomas: Racial difference in severity, symptoms and age at diagnosis. J Reprod Med .1996; 41 : 483-90.

6. Wallach EE, VlahouaNF. Uterine myoma : An overview of development, clinical features and management. Obstet Gynaecol 2004 ; 104 : 393406.

7. Perera ww, Senanayake H. Benign neoplasms of the Genital tract. Obstetric and Gynaecology for postgraduates; Ratnam SS, Bhasker Rao K, Arulkumaran S(ed ); orient Longman limited; Madras, India 1994; 426-28.

8. CarisonKJ, MillerBA, FowlerF jr. The Maine womens' health study. Outcome of hysterectomy. Obstet Gynaecol 1994; 83 : 556-75.

9. Kjeruff KH, Langeubern PW, Rhodes JC, Harvey LA et al . Effectiveness of hysterectomy . Obstet Gynaecol $2000 ; 95: 319-26$.

10. Fernandez H, Setrioui O, Virelizier C,et al. Hysteroscopic resection of submucosal in patients with infertility. Hum Reprod 2001; 16 : 1489-92.

11. Campo S, Campo V, Gambadauro P. Reproductive outcome before and after laparoscopic or abdominal myomectomy for subserous or intramural myomas. Eur J Obstet Gynaecol Reprod Biol 2003; 110: 215-19.

12. KhanamRA, KhatunN. Uterine fibromyoma: Profile of Bangladeshi women. The Journal of Obstetrics and Gynaecology. 2000: 1(1): 19-21.

13. Hosne Ara B. Management of leiomyoma of uterus; FCPS dissertation Dhaka 1991

14. Khan M. Clinical presentation and management of leiomyoma of uterus. A study of 50 cases; FCPS dissertation Dhaka 1997.

15. Emembulo Jo. Uterine fibromyomata presentation and management in Northern Nigeria. Int J Obstet. Gynaecol 1987; 25: 413- 16.

16. Buttrum VC, Reiter RC. Uterine leiomyomata . Etiology, Symptomatology and management. Fertility and sterility 1981; 36(4): 433- 45.

17. Muram D, Walter JH . Myoma of uterus in pregnancy: ultrasonographic follow up . Am J Obstet.Gynaecol 1980; 138: 16-19.

18. Stewart E A, Nowak R A . Leiomyoma - related bleeding: a classic hypothesis update for molecular era. Hum Reprod update 1996; 2: 296 306.

19. Propst AM, Hill JF $3^{\text {rd }}$. Anatomic factors associated with recurrent pregnancy loss. Smin Reprod Med 2000; 18: 341-50.

20. Parker WH, Fu YS, Berek JS. Uterine sarcoma in patients operated on for presumed leiomyoma and rapidly growing leiomyoma. Obstet Gynecol 1994; 83:414-18.

21. Dubbisson JB, Chave FX, Chapron C et al. Uterine rupture during pregnangy after laparoscopic myomectomy. Hum Reprod 1995; 70: 1475 -77.

22. lhakar R, Ayer S, Clarkson P, Staton S, Manyonda L. Outcomes after hysterectomy total versus subtotal abdominal hysterectomy. N Eng J Med 2002; 347: 1318- 25 . 